\title{
Personal genomes and precision medicine
}

\author{
Gareth Highnam and David Mittelman,2*
}

\begin{abstract}
A report of the fifth annual Personal Genomes and Medical Genomics meeting, held at Cold Spring Harbor Laboratory, Cold Spring Harbor, New York, USA, November 14-17, 2012.

Keywords high-throughput sequencing, personal genomics, personalized medicine, pharmacogenomics, precision medicine
\end{abstract}

Less than 10 years ago, the Sanger sequencing method provided the only technology for DNA sequencing. Highthroughput sequencing, also referred to as next-generation sequencing, changed the field by addressing limitations associated with conventional sequencing, such as throughput, scalability and cost. The first Personal Genomes meeting, held in 2008, was a forum for discussing some of the first personal genomes - namely, James Watson's genome, which was sequenced using highthroughput sequencing technology. Since then, the Personal Genomes meeting has expanded each year, reflecting the growing interest in exploiting genome technology to improve the diagnosis and treatment of human disease. This year, the fifth annual meeting reflected this interest by including pharmacogenomics and updated the meeting title to include medical genomics. The meeting covered innovations in the use of genomics for diagnosing Mendelian disorders and cancer and augmenting their treatment, as well as the technical, ethical and policy-based implications of transiting genome technology and genomic data in the clinic.

\section{Continued successes and challenges of clinical sequencing}

The first keynote speaker, Retta Beery (Encinitas, California, USA), presented her family's personal journey with twins suffering from Segawa's dystonia, a progressive muscle disorder that causes muscle spasms and impaired

*Correspondence: david.mittelman@vt.edu
'Virginia Bioinformatics Institute, Virginia Tech, Blacksburg, VA 24061, USA
Department of Biological Sciences, Virginia Tech, Blacksburg, VA 24061, USA motor control. The standard of care ( $\mathrm{L}$-dopa supplementation) was not fully effective, so, through whole-genome sequencing (WGS) by Baylor College of Medicine's Human Genome Sequencing Center, an improved, personalized treatment plan for the twins was established. This resulted in a miraculous improvement in their muscle control and in their quality of life. Beery went so far as to request that the names of the twins be included in publications to personalize the impact of this study, and stressed that her children are outstanding examples of the potential that genomics holds for medicine.

This success story, along with the growing number of other genome sequencing case studies, forms a cogent argument for integrating WGS in the clinic. This year, speakers shared stories of both the successes and challenges of this transition. For example, Stephen Quake (Stanford University, USA) shared his work on developing a non-invasive, prenatal test for Down syndrome, based on the high-throughput sequencing of fetal cell-free DNA from the whole blood of the mother. Quake pointed out that this is one of the first high-throughput sequencing tests to transition to the clinic.

Howard Jacob (Medical College of Wisconsin, Children's Hospital of Wisconsin, USA) outlined key challenges in clinical sequencing. While data storage, analysis and clinical certifications such as Clinical Laboratory Improvement Amendments (CLIA) or College of American Pathologists (CAP) remain costly challenges, Jacob argued that new challenges his group and others are facing involve reconciling genome analysis with the decision-making guidelines of physicians. To this effect, Jacob shared the story of a child with early onset, aggressive inflammatory bowel disease, where conventional testing rendered no clear diagnosis. Sequencing the child's genome revealed a rare variant in X-linked inhibitor of apoptosis (XIAP), and previous literature had connected XIAP to the inflammatory response and to the nucleotide-binding oligomerization domain (NOD) signaling pathway. However, to convince the physician to make a diagnosis and initiate treatment, further assays and experimental support were required. Convinced by this additional experimental evidence, the physician made the diagnosis and ordered a bone marrow transplant of cord blood cells. The patient recovered, and three years later has not experienced a recurrence of symptoms. 
Benchmarking the performance of clinical exomes Not all sequencing data result in clear, actionable information, as Jacob and other speakers point out. Certain portions of the genome are inherently inaccessible to some platforms and sequencing applications. Even when it is possible to detect genome variants, this does not necessarily lead to a change in treatment. Many speakers argued for exome sequencing, which is cost-effective and offers a similar or even better rate of actionable diagnoses compared with WGS. Sharon Plon (Baylor College of Medicine, USA) described the one-year status of clinical exome sequencing performed by Baylor College of Medicine's Whole Genome Laboratory (WGL), which also includes the mitochondrial genome. The WGL performs several quality control steps, as well as a consultation with a sign-out team of American Board of Medical Genetics-certified lab directors, medical directors, clinicians and genetic counselors, before releasing a two-part report on discovered exome variants. First, the focused variant report, which describes variants of known and unknown significance found in genes related to the reported disease or clinical phenotypes, is created and delivered directly to the requesting physician. A second, optional variant report is available upon request, and contains additional variants with no direct connection to the reported disease, or to clinical phenotypes. This clinical service has been applied to hundreds of exomes - of which $85 \%$ are from pediatric patients - with a successful diagnostic rate of about $25 \%$. The majority of exomes sequenced at the WGL are ordered by physicians for patients suffering from neurological phenotypes, for whom conventional genetic testing has yielded no insights.

Han Brunner (University Medical Center Nijmegen, the Netherlands) reported a similar success rate for diagnostic exome sequencing, and, like the WGL, he reported that the greatest portion of patients suffered from neurological disorders. Brunner also noted successful diagnoses even in disorders with only a single de novo mutation. For example, in two boys exhibiting nearly identical craniofacial phenotypes, Brunner found a common de novo mutation using exome sequencing. This phosphofurin acidic cluster sorting protein 1 (PACS1) mutation was then engineered into an animal model, and its causative relationship with the phenotype was verified. Lisenka Vissers (University Medical Center Nijmegen, Netherlands) also discussed the utility of exome sequencing in identifying de novo mutations in the majority of diagnosed intellectual disorders.

\section{Developing standards for analysis and interpretation}

There are several high-throughput sequencing platforms, with many emerging applications for sequencing. These platforms and applications offer different trade-offs of cost, speed, throughput, read lengths, error rates and bias. Ultimately, as these technologies mature, sequence analysis will be more straightforward, but in the meantime analysis remains a challenge and bottleneck.

Michael Eberle (Illumina, San Diego, USA) discussed the Illumina's Platinum Genome project, which aims to provide a gold standard set of genomes and variant calls for use in benchmarking sequence analysis tools. As Eberle explained, variant callers are often benchmarked by testing the Mendelian consistency of calls within a parent-offspring trio; however, this consistency test captures only a subset of genotyping error. Illumina has sequenced an extended family, leveraging a multigenerational pedigree to create a high quality set of wholegenome variant calls for the highly characterized CEPH/ Utah Pedigree 1463. Eberle reports that for singlenucleotide polymorphisms (SNPs), the set of variants is more than 99.9998\% accurate. As supporting data are gathered for every variant, the Platinum Genomes project will publicly release and curate the dataset.

There are also problems with variant calling that are rooted in short read sequencing technology, as Jay Shendure (University of Washington, USA) points out. Shendure proposes haplotype phasing as a means to improve variant calls. His method for a haplotyperesolved genome combines high-throughput sequencing with assemblies of barcoded fosmid libraries, each containing a small portion of the diploid genome.

The importance of ethnically diverse reference resources was also discussed at the meeting. For example, Jun Yang (St Jude Children Research Hospital, USA) found that racial differences among pediatric patients with acute lymphoblastic leukemia significantly affected susceptibility and outcome. Yang's study, involving more than 2,000 children with acute lymphoblastic leukemia, identified multiple treatment-affecting variants both specific and common to their different human subpopulations.

\section{Defining guidelines for sharing genomic data}

Anita Allen (Presidential Commission for the Study of Bioethical Issues (PCSBI), USA), described suggestions by the PCSBI for ensuring the ongoing privacy and security of data in the rapidly developing field of highthroughput sequencing. Allen stressed that although many variants are of unknown significance today, future discoveries may illuminate their significance. Therefore, it is important to develop policies to define how and when to share genetic information, even if it is not immediately actionable. Arthur Beaudet (Baylor College of Medicine, USA) also offered a perspective, as part of the ethics panel, on genetic counseling in prenatal diagnostics. Beaudet cites the Ashkenazi Jewish community as among the most progressive adopters of prenatal diagnostics, in spite of their sensitivity to genocide and 
eugenics. Gholson Lyon (Cold Spring Harbor Laboratory, USA) highlighted the need for improved variant calling and interpretation. In one example, he showed the disagreement of variant callers using a telling Venn diagram, referring to it as the three-ring circus. With regard to interpretation, Lyon pointed out challenges beyond improved annotation of variants - namely, penetrance and variable expressivity. The effect of variants on phenotype depends on the genetic background, and for this reason he argues for the analysis and interpretation of variants using extended families.

The final keynote lecture was delivered by Maynard Olson (University of Washington, USA), who urged the group to consider the term 'precision medicine' in place of 'personalized medicine.' The rationale is that highthroughput sequencing is enabling the precise diagnosis and treatment of disease. Olson further challenged genome scientists and institutions to focus on even larger scale populations to aid in the discovery of rare variants. Data sharing is also critical for advancement, Olson argued, and his idea for data sharing involved a large databank of anonymized, publicly shared data from which everyone may derive insight. The term 'precision medicine' was a hit at the meeting and is incorporated in the title for the upcoming 2013 meeting, 'Precision Medicine: Personal Genomes and Pharmacogenomics'.

\section{Conclusions}

Improvements in high-throughput sequencing technologies have made sequencing personal genomes feasible.
Case studies presented at the Personal Genomes and Medical Genomics meeting continue to demonstrate that clinical sequencing can be a powerful tool for the diagnosis and treatment of a variety of human diseases. However, challenges remain in fully characterizing variation in human genomes. Some of these challenges will resolve as sequencing technologies mature and analysis standards are better defined. As we commoditize the analysis of personal genomes, new challenges will center on interpreting the clinical impact of genomic data within the context of clan genomics, molecular patient profiles, medical records, and the scientific literature.

\section{Acknowledgements}

The authors would like to thank R Matthew Ward and members of the Mittelman Lab for helpful comments and suggestions. This work was supported by an R21 grant (NS079926) from the NIH to DM.

\section{Abbreviations}

CAP, College of American Pathologists; CLIA, Clinical Laboratory Improvement Amendments; WGL, Whole Genome Laboratory; WGS, whole-genome sequencing.

\section{Competing interests}

The authors declare that they have no competing interests.

Published: 19 December 2012

doi:10.1186/gb-2012-13-12-324

Cite this article as: Highnam G, Mittelman D: Personal genomes and precision medicine. Genome Biology 2012, 13:324. 